Communications in Physics, Vol.21, No. 1 (2011), pp. 35-41

\title{
A SMALL-ANGLE NEUTRON-SCATTERING STUDY IN Fe-Pt-Al
}

\author{
PHAM DUC THANG \\ Faculty of Engineering Physics and Nanotechnology, \\ University of Engineering and Technology, Vietnam National University, Hanoi
}

\begin{abstract}
In this paper a study on small-angle neutron-scattering (SANS) of the Fe-Pt-Al alloys was presented. The scattering intensities in an external magnetic field of $1.2 \mathrm{~T}$, perpendicular to the neutron-beam direction, are significantly smaller than these in the initial state. The experimental data were analyzed by means of the monodisperse and polydisperse models, depending on the sample state. The correlation between the derived results and other microstructure analyses will also be discussed.
\end{abstract}

\section{INTRODUCTION}

In Fe-Pt alloys, high-performance hard magnetic properties are connected with the fcc-fct structural phase-transition $[1,2]$. By adding a small amount of a third element, such as $\mathrm{Cu}, \mathrm{Zr}, \mathrm{Nb}$, etc. the magnetic properties of Fe-Pt alloys can be further improved. To investigate the change in microstructure during the phase transition, transmission electron microscopy (TEM) has been employed. TEM technique, however, only allows to study a small surface of the order of several $\mu \mathrm{m}^{2}$, whereas with small-angle neutron scattering (SANS) one can investigate not only the whole surface of the sample but also the bulk properties of nanometer-sized systems. Moreover, in the present of an applied magnetic field, SANS data include the scattering from the nuclei and from magnetic contributions. Therefore, by analysing the data, one can obtain information on the size of the particles and the magnetic domains of the phases in the sample.

This paper aims to analyze the SANS data, measured on the Fe-Pt-Al alloy, by using some different theoretical models, namely monodisperse and polydisperse models. The obtained results will be discussed in connection with other characterizations.

\section{EXPERIMENTAL AND THE MODELS}

$\mathrm{Fe}_{59.85} \mathrm{Pt}_{39.9} \mathrm{Al}_{0.25}$ alloys were prepared by arc-meltins appropriate amounts of pure ingots $(3 \mathrm{~N})$ of $\mathrm{Fe}, \mathrm{Pt}$ and $\mathrm{Al}$ under e purified-argon atmosphere. The melts were cast and then cut into disk-shaped samples with a diameter of $10 \mathrm{~mm}$ and about $1 \mathrm{~mm}$ thick for the SANS measurements. These samplas were firstly annealed at $1325{ }^{\circ} \mathrm{C}$ for $1 \mathrm{~h}$ under argon atmosphere and quenched in water, foilowed by an ageing process at $525{ }^{\circ} \mathrm{C}$ for 24 $\mathrm{h}$. Thlg process was also terminated by quenching in water.

The SANS experiments were carried out on the instrument D11 at ths ILL (Grenoble, France). The samples were measured at room temperature and in an applied tagnetic 
PHAM DUC THANG

field of up to $1.2 \mathrm{~T}$, perpendicular to the neutron-beam direction. The neutron wavelength was $1 \mathrm{~nm}$ with a wavelength spread of $10 \%$. The measurements were carried out in three different statee: before applying the magnetic field (the virgin state), in the magnetic field (the field state) and after removing the magnetic field (the remanent state). On the raw data, a background correction was performed using an empty $\mathrm{Cd}$ slit. The corrected data were calibrated absolutely by measurements of a wamer standard.

For analysing the SANS data, the two-phase model is one of the moht commonly used approximations in small-angle scattering. In this model, one assumes that the microstructure ef a particular system can be described by a collection of particles with uniform scattering-longth density $\rho_{p}$, embedded in a matrix wits scattering length density $\rho_{m}$. By using this assumption several important relations can be derived as presented in the following sections.

\section{II.1. The Porod law}

In the high-scattering vector $Q$ tail region of the scattering intensity $I(Q)$ curve, the Porod law can be used for a two-phasf system oe particles with smooth surfaces and sharp boundaries [3]:

$$
I(Q)=K_{P} Q^{-\alpha}+B
$$

with $K_{P}$ the Porod conltant, the exponent $\alpha$ which usually eqtals 4 , and $B$ a $Q$-independent (incoherent) background parameuer. This constant is related to the total surface area per unit vosume $S_{V}$ of the particles by:

$$
K_{P}=2 \pi(\Delta \rho)^{2} S_{V}
$$

where $\Delta \rho=\rho_{p}-\rho_{m}$ is the contrast in scattering-length density bdtween the particle $\left(\rho_{p}\right)$ ane the matrix $\left(\rho_{m}\right)$.

\section{II.2. Fouroer transfirmation}

For an ensemble oi randomly distributed scattering objects, the scattering intensfty depends only on the magnitude $Q$ and cen be axpressed as [4]:

$$
I(Q)=4 \pi N \int_{0}^{\infty} \frac{\sin (Q r)}{Q r} p(r) d r
$$

where $N$ is the surface density and $p(r)=\gamma(r) r^{2}$ is the pair distance distribution function, $\gamma(r)$ is the correlation function.

Due to the experimental limitations, for example the limited $Q$ range as mentioned above, the determinatcon of $p(r)$ by using a direct Fourier transform may lead to appreciable systematic errors. To solve this problem the indirect Fourier transform is most wpdely used to determine $p(r)$ then $I(Q)[5,6]$. If a system can be considered as monodisperse, the distance distribution function $p(r)$ can be determined from the scattering intensity calculation. The maximum correlation distance $D_{\max }$ which is rilated to the magnetic domain size can be obtained from this function. In the case of a polydisperse system [7], where particles may have different shapes, sezes or compositions, the size/volume distribution funition $D(R)$ can be determined. The average iarticle size is then derived from the $D(R)$ curve. 


\section{II.3. The Porod invariant}

For a two-phase system, if the scattering intensity is determined over a large $Q$ range, the Porod insariant iv given by

$$
Q_{P}=\int_{0}^{\infty} I(Q) Q^{2} d Q=2 \pi^{2}(\Delta \rho)^{2}
$$

The Porod invariant [3] is an important quantity because it represents the mean-square fluctuation of the system, which is insensitive to structural features of the scattering objects. Its value cin be obtained directly by integrating the $I(Q)$ curve. A spherical particle with radius $R$ has a total surface area per unat volume $S_{V}=S / V=3 / R$. Combining with Eq. (2) the average radius of spherical particles equals

$$
R=\frac{3}{\pi} \frac{Q_{P}}{K_{P}}
$$

\section{RESULTS AND DISCUSSION}

The SANS data of as-quenched and aged $\mathrm{Fe}_{59.85} \mathrm{Pt}_{39.9} \mathrm{Al}_{0.25}$ are presented in Fig. 1. Detailed results will be presented in the following sections. In general, SANS data is the total contribution from the nuclear and magnetic scattering. For the considered alloys, the scattering intensities in the field and remanent states are significantly smaller than in the virgin state. Therefore, in the virgin state the scattering contribution from randomly oriented magnetic domains is dominant. However, the SANS data in the field state are characterized by an inhomogeneous magnetization. Thus, in this state, the scattering from particles with different magnetization becomes the main contribution. For the remanent state, the analysis is more complicated and not very reliable due to significant contributions from all parts.

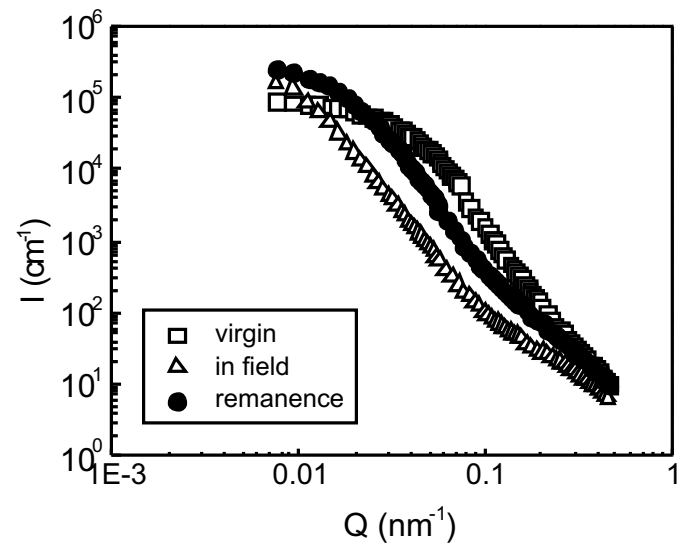

(a)

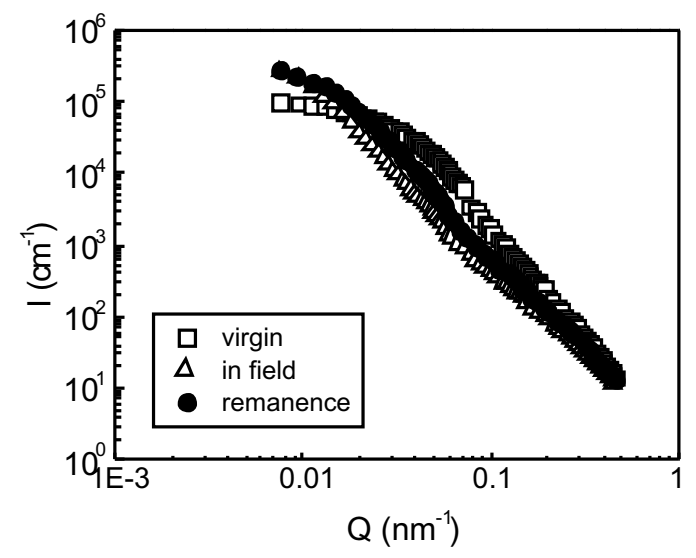

(b)

Fig. 1. Q dependence of the SANS intensities for as-quenched and aged $\mathrm{Fe}_{59.85} \mathrm{Pt}_{39.9} \mathrm{Al}_{0.25}$. 


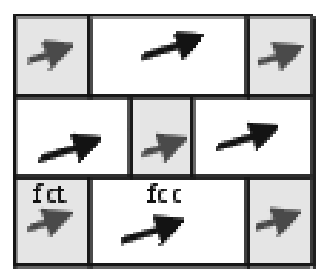

i-th domain

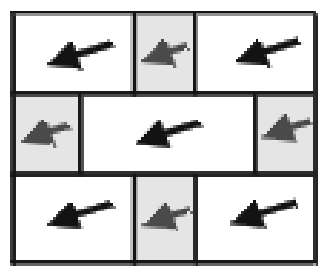

j-th domain

(a)

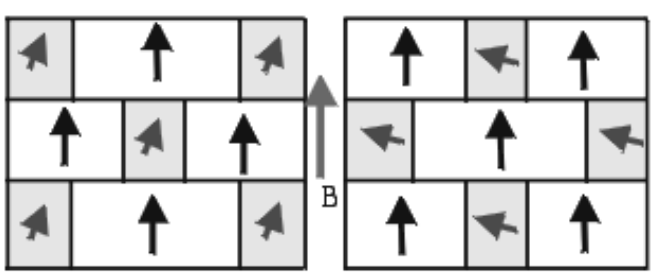

i-th domain j-th domain

(b)

Fig. 2. Structural aodel of mmgnetic domains and fcc and fct particles in Fe-tt alloys: (a) the virgin state, (b) Phe field state.

In Fig. 2 we present a structure model which assuming that the magnetic-domain size is larger than the fcc and fct particle sizes. In the virgin state, one may expect that the magnetic domains are randomly oriented and approximately of the same size. Let us simply indicate the fcc and fct particles as alternating particles in each domain. In the virgin state, the magnetic moments of the fcc and fct particles are parallel within each domain. The SANS, therefore, has main contributions from randomly oriented magnetic $i$-th, $j$-th, etc. domains in the whole sample (Fig. 2a). However, when a magnetic field is applied, the magnetic moments of the soft-magnetic fcc particles are more easily oriented along the magnetic field direction than those of the hard-magnetic fct particles (see Fig. $2 \mathrm{~b})$. In this case, the scattering on the fcc and fct particles, which have different magnetization directions, is dominant. To analyse the SANS data, we therefore propose that thg monodisperse model is suitable for the Fe-Pt alloys in the virgin state whereas the polydisperse model is necessary for the fteld state.

The Porod law was apppied in the high- $Q$ regions of the $I(Q)$ curve of aged $F_{59.85} \mathrm{Pt}_{39.9} \mathrm{Al}_{0.25}$. The parameters ottained from the Porod fitting are listed in Table 1 . In the analysis, the Porod exponent $\alpha$ is considered as an adjustable parameter. The parameter $K_{P}$ of the aged samples will be used latter to calculate the particle.

Table 1. Parameters obtained from the Porod law for aged $\mathrm{Fe}_{59.85} \mathrm{Pt}_{39.9} \mathrm{Al}_{0.25}$.

\begin{tabular}{|ccccc|}
\hline State & $10^{9} K_{P}\left(\mathrm{~nm}^{-1-\alpha}\right)$ & $\mathrm{B}\left(\mathrm{cm}^{-1}\right)$ & $\alpha$ & $Q$ range $(\mathrm{nm})$ \\
\hline virgin & $291.6 \pm 12.7$ & $-7.9 \pm 0.7$ & $2.60 \pm 0.03$ & $0.2<Q \leq Q_{\max }$ \\
\hline field & $227.3 \pm 51.9$ & $-3.3 \pm 0.8$ & $2.36 \pm 0.15$ & $0.25<Q \leq Q_{\max }$ \\
\hline
\end{tabular}

Let us consider that all $\mathrm{Fe}_{59.85} \mathrm{Pt}_{39.9} \mathrm{Al}_{0.25}$ are monodisperse systems. The SANS data of the as-quenched and aged samples in the virgin state and the fitting results by means of the GNOM program are presented in Fig. 3, together with the fitting results. The experiments and calculations are in goom agreement. The parameters obtained from the fitting are presented in Table 2. The distance distribution functions are plotted in Fig. 4 for as-quenched and aged $\mathrm{Fe}_{59.85} \mathrm{Pt}_{39.9} \mathrm{Al}_{0.25}$. The magnetic-domain size as derived frod the virgin state is about $155 \mathrm{~nm}$ for as-quenched $\mathrm{Fe}_{59.85} \mathrm{Pt}_{39.9} \mathrm{Al}_{0.25}$ and about $140 \mathrm{~nm}$ for aged $\mathrm{Fe}_{59.85} \mathrm{Pt}_{39.9} \mathrm{Al}_{0.25}$. 


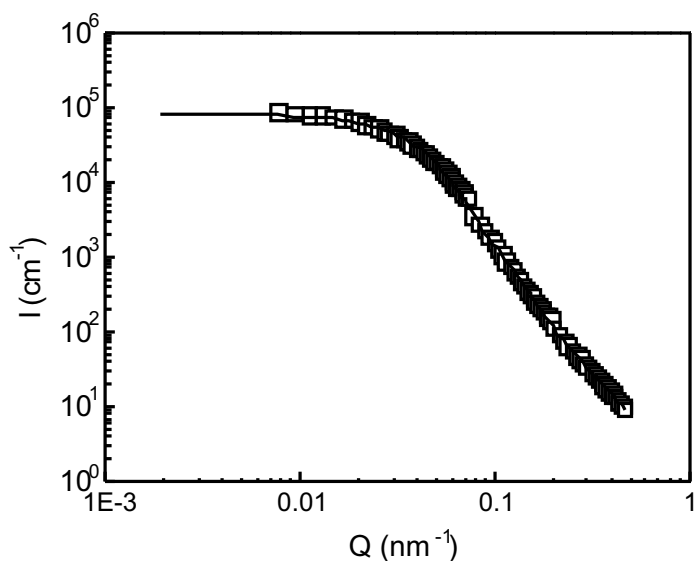

(a)

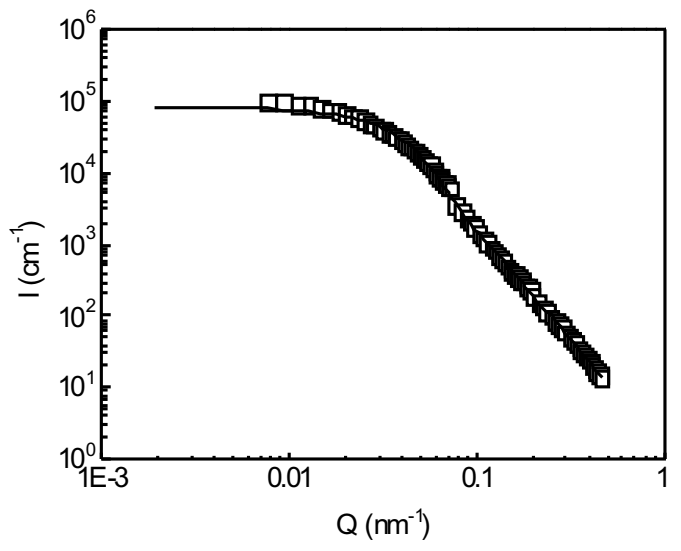

(b)

Fig. 3. Experimental and calcualted $\mathrm{Q}$ dependence of the SANS intensity for as-quenched (a) and aged (b) $\mathrm{Fe}_{59.85} \mathrm{Pt}_{39.9} \mathrm{Al}_{0.25}$.

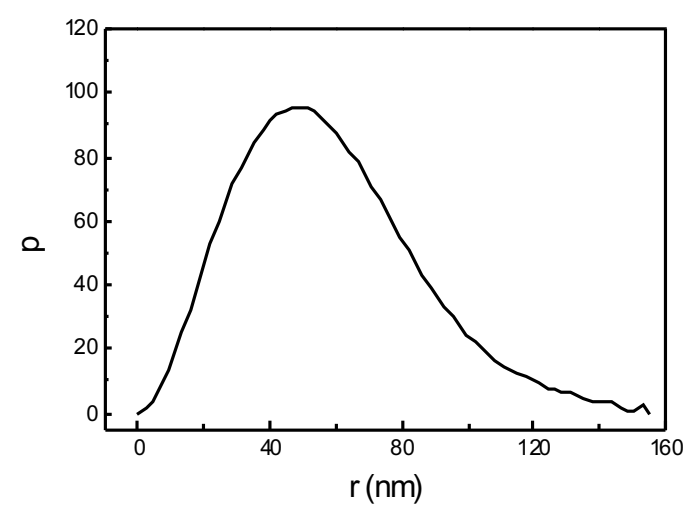

(a)

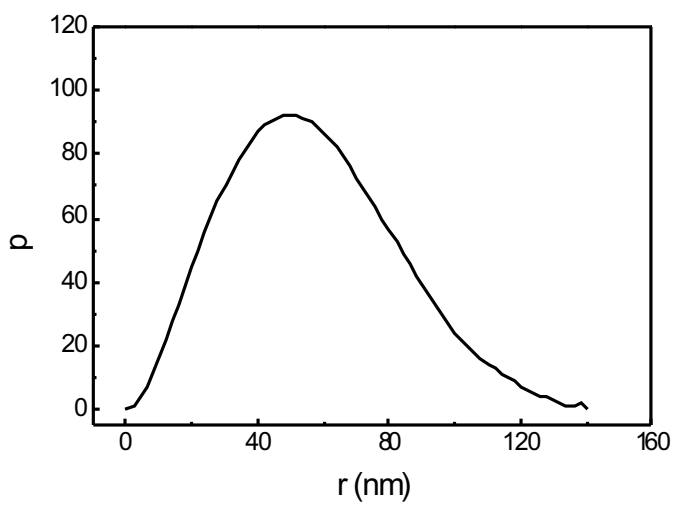

(b)

Fig. 4. Distance distribution function $\mathrm{p}(\mathrm{e})$ for as-quenchrd (a) and aged (b) $\mathrm{Fe}_{59.85} \mathrm{Pt}_{39.9} \mathrm{Al}_{0.25}$.

Table 2. Fitting patameters for the virgin state of $\mathrm{Fe}_{59.85} \mathrm{Pt}_{39.9} \mathrm{Al}_{0.25}$.

\begin{tabular}{|l|l|l|l|}
\hline Sample & $D_{\max }(\mathrm{nm})$ & $R_{g}(\mathrm{~nm})$ & $I_{0}\left(10^{4} \mathrm{~cm}^{-1}\right)$ \\
\hline as-quenched & 155 & $45.0 \pm 0.1$ & $8.0 \pm 0.02$ \\
\hline aged & 140 & $44.0 \pm 0.1$ & $7.7 \pm 0.02$ \\
\hline
\end{tabular}

We will now use the polydisperse model to analyse the SANS data of the $\mathrm{Fe}_{59.85} \mathrm{Pt}_{39.9} \mathrm{Al}_{0.25}$ alloys. In Fig. 5, we present the experimental data and the calculations for aged $\mathrm{Fe}_{59.85} \mathrm{Pt}_{39.9} \mathrm{Al}_{0.25}$. The fits are in reasonable agreement with the experimental data. It should be noted that, 


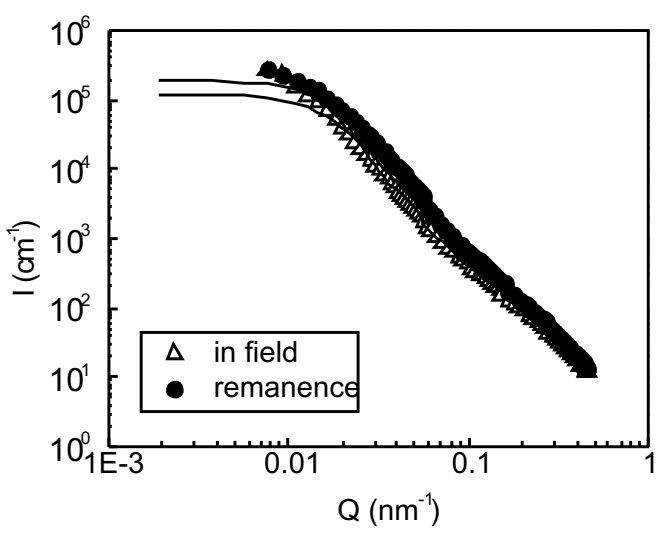

Fig. 5. Calculatod Q dependence of the SANS intensities fer aged $\mathrm{Fe}_{59.85} \mathrm{Pt}_{39.9} \mathrm{Al}_{0.25}$.

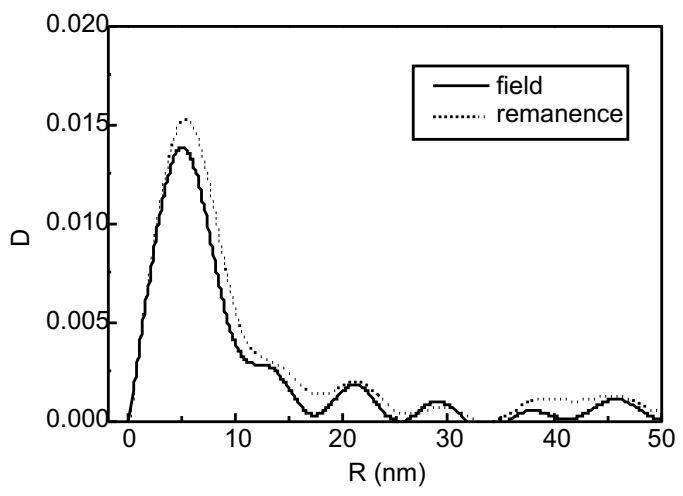

Fig. 6. Volume distributiln function $\mathrm{D}(\mathrm{R})$ for aged $\mathrm{Fe}_{59.85} \mathrm{Pt}_{39.9} \mathrm{Ao}_{0.25}$.

Table 3. Fittilg parameFers for aged te ${ }_{59.85} \mathrm{Pt}_{39.9} \mathrm{Al}_{0.25}$.

\begin{tabular}{|l|c|c|c|}
\hline State & $R_{\max }(\mathrm{nm})$ & $R_{g}(\mathrm{~nm})$ & $I_{0}\left(10^{4} \mathrm{~cm}^{-1}\right)$ \\
\hline field & 130 & $90.5 \pm 0.1$ & $12.3 \pm 0.1$ \\
\hline remanent & 125 & $84.3 ? 0.1$ & $18.9 ? 0.1$ \\
\hline
\end{tabular}

using this model, we only focus on the SANS data in the field state. The data of asquenched $\mathrm{Fe}_{59.85} \mathrm{Pt}_{39.9} \mathrm{Al}_{0.25}$ cannot be fitted. The parameters and the volume distribution functions of the spherical particles obtained from the fitting are presented in Table 3 (where $R_{\max }$ is the maximum radius of the particles) and in Fig. 6. In the field state, the $D(R)$ curve displays a maximum at $R \approx 4 \mathrm{~nm}$. There are two other small peaks around 12 and $22 \mathrm{~nm}$. The main peak at about $4 \mathrm{~nm}$ correlates with the fine fct nano-particles with average size of 3-7 nm, as can be seen in the TEM images [8]. Furthermore, from Eq. (5), by using the Porod invariant $Q_{P}$ and the Porod constant $K_{P}$, the size of the fct particles of aged $\mathrm{Fe}_{59.85} \mathrm{Pt}_{39.9} \mathrm{Al}_{0.25}$ can be estimated as in Table 4 . The value of about 3.5 
nm agrees well with the result obtained by TEM analysis, which provides sizes of about 3-7 nm.

Table 4. The fct particle sizes for aged $\mathrm{Fe}_{59.85} \mathrm{Pt}_{39.9} \mathrm{rl}_{0.25}$.

\begin{tabular}{|l|c|c|c|}
\hline State & $Q_{P}\left(10^{-7} \mathrm{~nm}^{-\alpha}\right)$ & $K_{P}\left(10^{-7} \mathrm{~nm}^{-1-\alpha}\right)$ & $2 R(\mathrm{~nm})$ \\
\hline field & 4.180 & 2.273 & 3.5 \\
\hline remanent & 5.885 & 3.420 & 3.3 \\
\hline
\end{tabular}

\section{CONCLUSIONS}

The scattering data of $\mathrm{Fe}_{59.85} \mathrm{Pt}_{39.9} \mathrm{Al}_{0.25}$ were analyoed by means of several methods. Firstly, thn Porod law is not only valid for an exponent value $\alpha=4$ but even for values smaller than 4 . This suggests non-smooth and non-sharp surfaces (boundaries) of (or between) the particles which may be coneected with lattice defects or an inhomogeneous distributisn of the elements [9].

The monodisperse model was applied to fit the SANS data of all samples in the virgin state. Information on the magnetic-domain size was obtained which is in the range of a few hundred nanometres. Meanwhile the polydisperse model was used for the SANS data in the field (and remanent) states to estimate average particle size of fct phase. This marticle size was also calculated from the Porod invariant. These are in good agreement with the results observed by other analysis methods $[8,10]$.

\section{REFERENCES}

[1] M. Hansen, ConstitGtion of Binary Alloys, Mcuraw Hill, New York, 1958, p. 698.

[2] K. Watanabe, Trans. Jpn. Inst. Met. 51 (1987) 91.

[3] G. Porod, Kolloid - Z 125 (1952) 51.

[4] A. Guinier nad a. Fournet, Small Angle Scattering of X-rGys, Wiley Publisher, New York, 1955.

[5] O. Glatter, J. Appl. Cryst. 10 (1977) 415.

[6] O. Glatter, J. Appl. Cryst. 13 (1980) 7.

[7] D.I. Svergun, J. Cpp. Aryst. 25 (1992) 495.

[8] P.D. Thang, E. Brück, F.D. Tichelaar, K.H.J. Buschow and F.R. de Boer, IEEE Trans. Magn. 38 (2002) 2934-2936.

[9] V.M. NaNutov, d.A. Bulavin and V.M. Garamus, Mater. Sci. and Engin. A264 (1999) 286.

[10] T. Klemmer, D. Hoydirk, H. Okumura, B. Zhang and W.A. Soffa, Scripta Metall. et Mater. 33 (1995) 1525.

Received 23 August 2010. 